\title{
Exploring brown dwarf disks
}

\author{
A. Natta and L. Testi \\ Osservatorio Astrofisico di Arcetri, Largo E. Fermi 5, 50125 Firenze, Italy \\ Received 3 July 2001 / Accepted 24 July 2001

\begin{abstract}
We discuss the spectral energy distribution of three very low mass objects in Chamaeleon I for which ground-based spectroscopy and photometry as well as ISO measurements in the mid-infrared are available (Comerón et al. 2000; Persi et al. 2000). One of these stars (Cha H $\alpha 1$ ) is a bona-fide brown dwarf, with mass $0.04-0.05 M_{\odot}$. We show that the observed emission is very well described by models of circumstellar disks identical to those associated to $\mathrm{T}$ Tauri stars, scaled down to keep the ratio of the disk-to-star mass constant and to the appropriate stellar parameters. This result provides a first indication that the formation mechanism of $\mathrm{T}$ Tauri stars (via core contraction and formation of an accretion disk) extends to objects in the brown dwarf mass range.
\end{abstract}

\section{Introduction}

The last few years have seen an enormous progress in our understanding of sub-stellar mass objects, as more and more objects in the mass range of brown dwarfs (BD; $\left.M_{\star} \lesssim 0.75 M_{\odot}\right)$ and giant planets $\left(\lesssim 0.015 M_{\odot}\right)$ are found, both in the field and in regions of recent star formation (Basri 2000; Lucas \& Roche 2000; Zapatero Osorio et al. 2000). It is now clear that "free-floating" very low mass objects exist. Do they form, as low-mass stars do, from the collapse of a molecular core? This, although apparently very reasonable, is not the only possibility. Alternative hypotheses have been suggested; for example that BD form in gravitationally unstable regions of protostellar disks (Pickett et al. 2000) or that they are stellar embryos, whose further growth is prevented by dynamical ejections from small stellar systems (Reipurth \& Clarke 2001).

A full understanding of the formation mechanism(s) of sub-stellar objects will take time. At present, it is timely to begin to explore the properties of BD systems in regions of star formation and compare them to the much better known pre-main-sequence low-mass stars, the T Tauri stars (TTS). The most important clue to a formation mechanism involving accretion from a parental core is the presence of a circumstellar disk. Claims of evidence of excess emission in the near (Oasa et al. 1999; Muench et al. 2001) and mid-IR (Comerón et al. 2000) in BD or BDcandidates in star-forming regions are beginning to appear in the literature. $\mathrm{H}_{\alpha}$ (Comerón et al. 1999; Comerón et al. 2000) and X-ray emission (Neuhauser \& Comerón 1998; Comerón et al. 2000) are seen in some very low mass objects. Muzerolle et al. (2000) detect evidence of magne-

Send offprint requests to: A. Natta,

e-mail: natta@arcetri.astro.it tospheric accretion at a very low rate $\sim 5 \times 10^{-12} M_{\odot} \mathrm{yr}^{-1}$ in the spectrum of the M 6 object V410 Anon 13, whose mass is estimated in the range $0.04-0.06 M_{\odot}$.

In very cold $\mathrm{BD}$, the most convincing evidence of excess emission, the accepted signature of circumstellar disks, can only be obtained in the mid-IR. There are at present very few bona-fide BD (i.e., stars with spectroscopical classification) with measured mid-IR excess. Comerón et al. (2000) list a small group of BD detected in the mid-IR survey of Cham I at 6.7 and $14.3 \mu \mathrm{m}$ by ISO (Persi et al. 2000). Of these, only three have detections in both bands, the one with the lowest mass being Cha $\mathrm{H} \alpha 1$, a M 7.5 star with mass $0.04-0.05 M_{\odot}$.

In this paper, we show that disk models analogous to those developed for TTS can well account for the observed spectral energy distribution (SED) of these three stars, providing strong support to the idea that BD form like the more massive TTS. We will also speculate somewhat on the derived disk properties, and on the possibility of extending this kind of study to objects of lower mass.

\section{Disk models}

We have computed the emission expected from a circumstellar disk heated by the irradiation of the central star following the method outlined by Chiang \& Goldreich (1997, CG97), which has been successfully applied to pre-mainsequence TTS and Herbig Ae stars (Natta et al. 2000a, 2001; Chiang et al. 2001). CG97 consider flared disks, in hydrostatic equilibrium in the vertical direction, and make a number of simplifying assumptions which permit the computation of the resulting SED in a quick and efficient fashion. Although not entirely self-consistent, such models 
provide a good first approximation to the SED, more than sufficient for the purpose of this paper.

We have taken for the various model parameters values typical of pre-main-sequence stars, scaled down where necessary. We have assumed that the circumstellar disk extends inwards to $R_{\star}$, has outer radius $R_{\mathrm{D}}=100 \mathrm{AU}$, total mass $M_{\mathrm{D}}=0.03 M_{\star}$ (Natta et al. 2000b), powerlaw surface density $\Sigma \propto R^{-1.5}$; the dust in the disk midplane has opacity $\kappa=0.01(1.3 \mathrm{~mm} / \lambda) \mathrm{cm}^{2} \mathrm{~g}^{-1}$ (Beckwith et al. 1990). On the disk surface, we assume the mixture of carbonaceous and silicate grains that provides a good fit to the mid-IR emission of pre-main-sequence stars (see Natta et al. 2001 for details). Most of these parameters are either irrelevant for the determination of the mid-IR flux, or appear in combinations, and cannot be individually constrained by the data available (see, for a discussion, Chiang et al. 2001). At this stage, only the most "standard" assumptions are justified.

The stellar properties have been determined by Comerón et al. (2000). The most important parameter for the disk SED calculation is the stellar luminosity and, to a lesser degree, the ratio $M_{\star} / R_{\star}$, that controls the disk flaring angle (roughly $\propto\left(R_{\star} / M_{\star}\right)^{4 / 7}$; CG97). We have used in displaying the results of our calculations the model stellar atmospheres of Allard et al. (2000, 2001). When comparing them with the broad-band visual and near-infrared photometry, we found good agreement only for values of the effective temperatures significantly lower than the spectroscopically determined values of Comerón et al. (2000). We do not know if this effect has any significance, given the uncertainties on the temperature scale in this mass range. In any case, our effective temperatures are well within the range of values expected for field brown dwarfs of similar spectral type (see, for example, Leggett et al. 2001), and their exact value is unimportant for the disk SED determination. The extinction and luminosity we derive are identical to the Comerón et al. values for Cha $\mathrm{H} \alpha 1$ and Cha $\mathrm{H} \alpha 2$, while they are definitely higher for Cha $\mathrm{H} \alpha 9$, for which we estimate a luminosity of $0.018 L_{\odot}$ as compared with Comerón et al. value of $0.0056 L_{\odot}$. The adopted values of the stellar parameters are given in the figure captions. We have not re-determined the stellar masses, for which we used the Comerón et al. estimates.

The most interesting of the three stars is Cha $\mathrm{H} \alpha 1$ which has the lowest mass of the three (about 0.04$\left.0.05 M_{\odot}\right)$. The results are shown in Fig. 1 . The disk predictions fit extremely well the observed points at all available wavelengths.

The results for the other two stars are shown in Figs. 2 and 3, respectively. Our standard disk model fits rather well the observed photometric points, although we note a tendency of the $14.3 \mu \mathrm{m}$ point to lie slightly below the model predictions. This discrepancy, however, is only $20 \%$ for Cha $\mathrm{H} \alpha 2$ and $25 \%(2 \sigma)$ for Cha $\mathrm{H} \alpha 9$. For this star, we show in Fig. 3 the predictions of the same disk model seen with an inclination angle of $75 \mathrm{deg}$ (dashed line). The flux is only slightly reduced, since it is dominated at

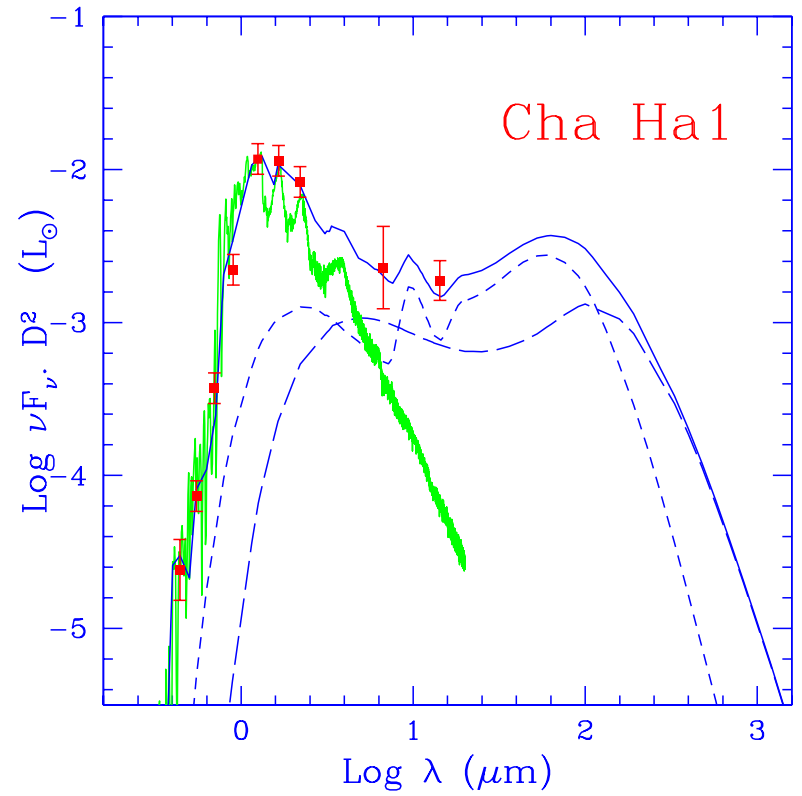

Fig. 1. SED of Cha H $\alpha 1$. The star has $T_{\star}=2400 \mathrm{~K}, L_{\star}=$ $0.01 L_{\odot}, M_{\star}=0.05 M_{\odot}$, extinction in the $I$ band $A_{I}=$ 0.11 mag. Disk parameters as in the text, the viewing angle is $0^{\circ}$ (face-on). The jagged line shows the Allard et al. (2001) model atmosphere, the solid line the disk model predictions. The short and long dashed lines show separately the contribution to the SED of the disk surface and midplane. The filled squares with error bars are the observed fluxes from Comerón et al. (2000).

most wavelengths by emission of optically thin material (see Figs. 1 and 2). The dot-dashed line shows the SED predicted by a geometrically flat disk seen face-on (dotdashed line). While the difference at long wavelengths is very large, the mid-infrared flux would still be consistent with the ISO measurements. One point worth to notice is that in these low luminosity objects the mir-IR is emitted by the inner disk, and the ISO points would not be consistent with a disk inner hole larger than about $3 R_{\star}$.

The results shown in Figs. 1, 2 and 3 provide good evidence that the same kind of disks which reproduce the properties of pre-main-sequence stars exist around lowermass objects, including a bona-fide BD such as Cha $\mathrm{H} \alpha 1$. In all three cases, the disks need to be optically thick in the mid-infrared. This, however, sets only a weak constraint on the mass of the disk, which remains optically thick at $14.3 \mu \mathrm{m}$ as long as $M_{\mathrm{D}} \gtrsim 1.5 \times 10^{-6} \kappa_{14.3 \mu \mathrm{m}} M_{\odot}$, where $\kappa_{14.3 \mu \mathrm{m}}$ is in units of $\mathrm{cm}^{2} \mathrm{~g}^{-1}$. For $\kappa_{14.3 \mu \mathrm{m}} \sim 3-10$ (Henning \& Stognienko 2000), this limit translates into $M_{\mathrm{D}} \gtrsim 10^{-5} M_{\odot}$, or $\gtrsim 2 \times 10^{-4} M_{\star}$, about ten times lower than the smallest measured ratios in pre-main-sequence stars (Natta et al. 2000b). The disk mass, and, to some extent, its size, can be better determined from millimeter data. For a typical value $M_{\mathrm{D}} \sim 0.03 M_{\star}$, we predict for Cha $\mathrm{H} \alpha 1$ a $1.3 \mathrm{~mm}$ flux of $3 \mathrm{mJy}$, well within the range of existing millimeter telescopes.

Cha $\mathrm{H} \alpha 1$ has spectral type $\mathrm{M} 7.5$ and mass of 0.04-0.05 $M_{\odot}$. Of course, one would like to extend our 


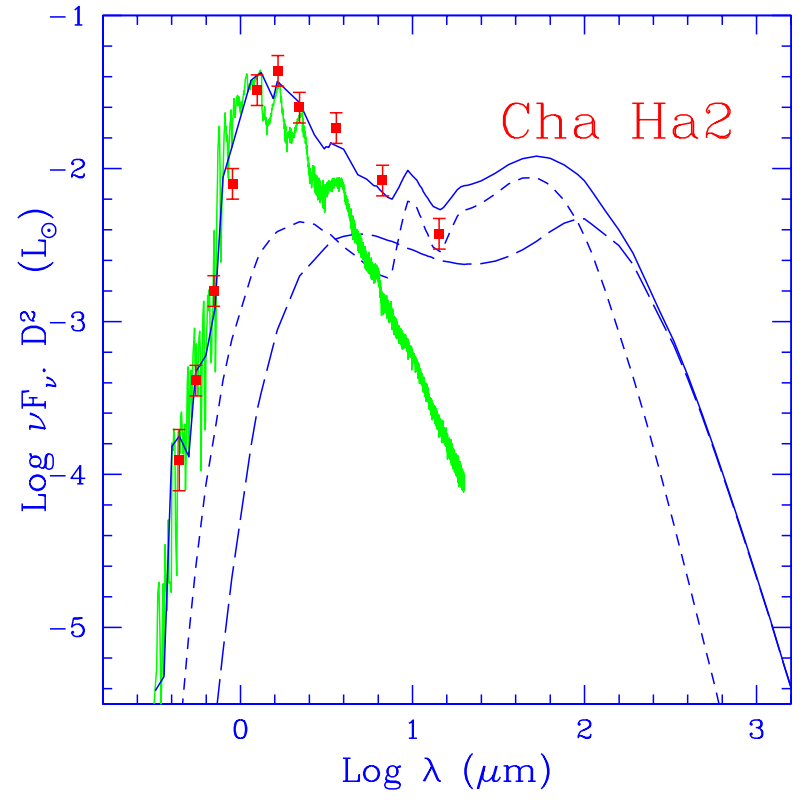

Fig. 2. Same as Fig. 1 for Cha $\mathrm{H} \alpha 2$. The star has $T_{\star}=2550 \mathrm{~K}$, $L_{\star}=0.035 L_{\odot}, M_{\star}=0.08 M_{\odot}, A_{I}=0.4 \mathrm{mag}$. The disk viewing angle is $0^{\circ}$. Note that the formal errors on the midinfrared fluxes quoted by Comerón et al. are $6 \%$ and $5 \%$ at 6.7 and $14.3 \mu \mathrm{m}$, respectively, while we have plotted error bars corresponding to a more realistic $10 \%$ uncertainties. The point at $3.6 \mu \mathrm{m}$ is from Kenyon \& Gómez (2000).

knowledge of circumstellar disks to even lower mass objects, such as are currently being discovered in the Orion Nebula Cluster (ONC; Lucas \& Roche 2000) and in $\sigma$ Orionis (Zapatero Osorio et al. 2000). Figure 4 shows the predicted SED for objects of lower and lower mass, as labelled, assuming an age of 2 Myr. Photospheric effective temperatures and luminosities are from Baraffe et al. (1998) and Burrows et al. (2001). Given the heuristic purpose of this figure, we have assumed that the stellar emission can be described by a black body at $T_{\star}$. This, as already noted, does not affect the calculations of the disk emission, but is a poor description of the photospheric spectrum. As a consequence, the SEDs in Fig. 4 are not realistic at wavelengths shorter than $\sim 4 \mu \mathrm{m}$, where the photospheric emission dominates.

Our calculations show that in nearby star forming regions it will be possible to detect disks around young sub-stellar objects with current instrumentation. Modern mid-infrared cameras at large telescopes have a $10 \mu \mathrm{m}$ sensitivity allowing detection of disk emission from systems more massive than $\sim 10 \mathrm{M}_{J}$. The upcoming space missions (SIRTF, HERSCHEL and especially NGST) will allow to detect disk emission around planetary-mass objects in the ONC and the $\sigma$ Orionis clusters. Dust spectroscopy in the mid-infrared, as obtained by ISO for luminous pre-main-sequence A stars (see Waelkens et al. 1996), is beyond the capability of even the largest groundbased telescopes, but for the most massive and nearby BD; in principle SIRTF and NGST will allow the observation of the spectra of lower mass or more distant objects,

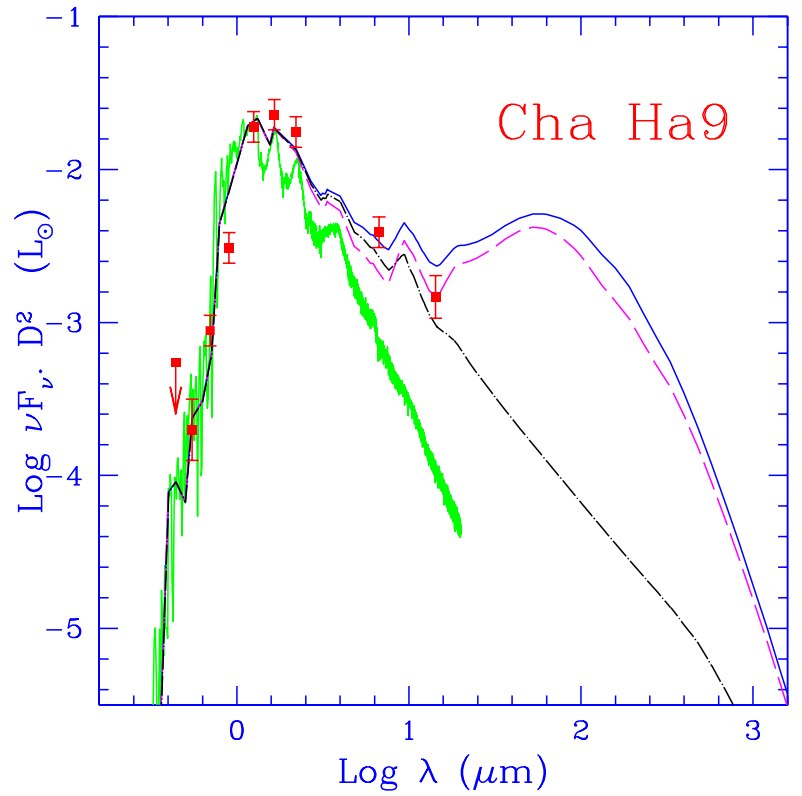

Fig. 3. Same as Fig. 1 for Cha $\mathrm{H} \alpha 9$. The star has $T_{\star}=2500 \mathrm{~K}$, $L_{\star}=0.018 L_{\odot}, M_{\star}=0.09 M_{\odot}, A_{I}=1.5 \mathrm{mag}$. The disk viewing angle (solid line) is $0^{\circ}$. Also shown are the predictions of the same disk seen at an inclination of $75^{\circ}$ (dashed line) and of a flat disk seen at $0^{\circ}$ (dot-dashed line). To avoid confusion, we do not show the disk surface and midplane constributions separately.

although the former satellite may be seriously affected by confusion problems. Perhaps the most interesting observations, determining the amount of circumstellar material around these objects and possibly probing the disk kinematics, will be those in the millimeter-wave range. As noted above, our disk model for Cha $\mathrm{H} \alpha 1$ predicts a continuum flux (about $3 \mathrm{mJy}$ at $1.3 \mathrm{~mm}$ ) well within the range of detectability with current instrumentation. To detect disks around less massive and more distant systems and to attempt the detection of molecular line emission in order to study the disk kinematics, we will need to wait for the ALMA array to be operational.

\section{Conclusions}

The presence and properties of disks around very low mass objects, which are being discovered in regions of star formation, are of crucial importance for understanding their formation. If disks exist, and if their properties are analogous to those of disks around TTS, we can conclude that the formation mechanism of TTS, by collapse and accretion of a molecular core, is very likely to extend to the lowest mass objects.

This paper presents the first attempt to test quantitatively this hypothesis. We have modeled the emission expected from disk models of properties identical to those of TTS for three objects in Chamaeleon I. These objects have been studied spectroscopically by Comerón et al. (2000), who attributed to them spectral types M7.5-M6 and masses in the range $0.04-0.09 M_{\odot}$. The lowest mass 


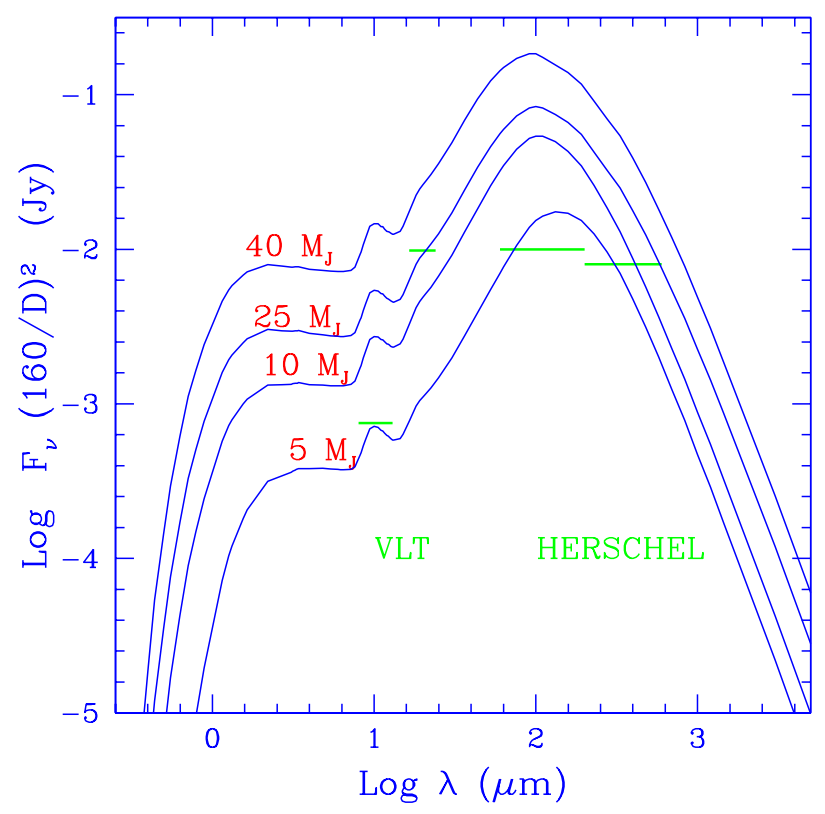

Fig. 4. Plot of the expected flux for BD of decreasing mass, from 40 Jupiter mass $\left(0.04 M_{\odot}\right)$ to $5 M_{J}$, as labelled. Below $\sim 4 \mu \mathrm{m}$, the emission is dominated by the stellar photosphere, assumed here as a black body at $T_{\star}$. The expected detection limit $(10 \sigma$ in $1 \mathrm{hr})$ are shown for the VLT/VISIR mid-infrared camera and for the far-infrared bolometers on HERSCHEL (horizontal bars).

object (Cha $\mathrm{H} \alpha 1)$ is certainly a bona-fide $\mathrm{BD}$. These objects are of particular importance because they have been detected at 6.7 and $14.3 \mu \mathrm{m}$ by ISO (Persi et al. 2000), providing a unique (until now) possibility to constrain the disk properties of BD. Inspection of Figs. 1 to 3 shows that it is extremely difficult to infer the existence of disks from near-infrared photometry, where the emission is largely dominated by the stellar photosphere.

In all three cases, and in particular in the most interesting object Cha $\mathrm{H} \alpha 1$, optically thick, flared disks are required to account for the mid-infrared fluxes. The disks are heated by the central star, and we expect that relatively small grains on the disk surface contribute significantly to the observed mid-infrared emission. If higher spectral resolution data in this range were available, we predict that one would observe the $10 \mu \mathrm{m}$ silicate feature in emission. Such disks are identical to those around TTS, just scaled to the appropriate stellar parameters.

This result seems to us very interesting. It provides a first indication that the TTS formation mechanism (from a collapsing core via an accretion disk) extends to objects of $0.04-0.05 M_{\odot}$. It does not, however, rule out the possibility that $\mathrm{BD}$ are ejected stellar embryos, as suggested by Reipurth \& Clarke (2001). In their model, the embryos may keep a small circumstellar disk, of few AU size. The existing data, limited to wavelengths shorter than $\sim 15 \mu \mathrm{m}$, can only set a limit to the disk radius of $R_{\mathrm{D}} \gtrsim 1 \mathrm{AU}$. There are two ways to proceed, the first and more obvious is to detect the millimeter emission of at least some $\mathrm{BD}$, which will be well below detection in the embryo hypothesis. The second is to search for other BD and planetary mass objects in the mid-infrared. A high frequency of disks is not predicted by the Reipurth \& Clarke model, since the small, truncated disks, associated with the embryos, not fed by any surrounding core, will rapidly disappear.

Acknowledgements. This work was partly supported by ASI grant ARS 1/R/27/00 to the Osservatorio di Arcetri.

\section{References}

Allard, F., Haushildt, P. H., \& Schweitzer, A. 2000, ApJ, 539, 366

Allard, F., Haushildt, P. H., Alexander, D. R., Tamanai, A., \& Schweitzer, A. 2001, ApJ, in press [astro-ph/0104256]

Baraffe, I., Chabrier, G., Allard, F., \& Hauschildt, P. H. 1998, A\&A, 337, 403

Basri, G. 2000, ARAA, 38, 485

Beckwith, S. V. W., Sargent, A. I., Chini, R. S., \& Guesten, R. 1990, AJ, 99, 924

Burrows, A., Hubbard, W. B., Lunine, J. I., \& Liebert, J. 2001, Rev. Mod. Phys., in press [astro-ph/0103383]

Chiang, E. I., \& Goldreich, P. 1997, ApJ, 490, 368

Chiang, E. I., Joung, M. K., Creech-Eakman, M. J., et al. 2001, ApJ, 547, 1077

Comerón, F., Rieke, G. H., \& Neuhäuser, R. 1999, A\&A, 343, 477

Comerón, F., Neuhäuser, R., \& Kaas, A. A. 2000, A\&A, 359, 269

Henning, Th., \& Stognienko, R. 1996, A\&A, 311, 291

Kenyon, S. J., \& Gómez, M. 2001, AJ, 121, 2673

Leggett, S. K., Allard, F., Geballe, T. R., Hauschildt, P. H., \& Schweitzer, A. 2001, ApJ, 548, 908

Lucas, P. W., \& Roche, P. F. 2000, MNRAS, 314, 858

Muench, A. A., Lada, E. A., Alves, J. A., \& Lada, C. J. 2001, AAS, 198.6907M

Muzerolle, J., Briceño, C., Calvet, N., et al. 2000, ApJ, 545, L141

Natta, A., Meyer, M. R., \& Beckwith, S. V. W. 2000a, ApJ, 534, 838

Natta, A., Grinin, V. P., \& Mannings, V. 2000b, in Protostars and Planets IV, ed. V. Mannings, A. P. Boss, \& S. S. Russell (Tucson: Univ. of Arizona Press), 559

Natta, A., Prusti, T., Neri, R., Wooden, D., \& Grinin, V. P. 2001, A\&A, 371, 186

Neuhäuser, R., \& Comerón, F. 1998, Science, 282, 83

Oasa, Y., Tamura, M., \& Sugitani, K. 1999, ApJ, 526, 336

Persi, P., Marenzi, A. R., Olofsson, G., et al. 2000, A\&A, 357, 219

Pickett, B. K., Durisen, R. H., Cassen, P., \& Mejia, A. C. 2000, ApJ, 540, L95

Reipurth, B., \& Clarke, C. J. 2001, AJ, 122, 432

Waelkens, C., Waters, L. B. F. M., de Graauw, M. S., et al. 1996, A\&A, 315, L245

Zapatero Osorio, M. R., Béjar, V. J. S., Martín, E. L., et al. 2000, Science, 290, 103 